\title{
Determination of cosmic-ray primary mass on an event-by-event basis using radio detection
}

\author{
Washington Carvalho $\mathrm{Jr}^{1{ }^{1, \star}}$ and Jaime Alvarez-Muñiz ${ }^{2}$ \\ ${ }^{1}$ Instituto de Física, Universidade de São Paulo, São Paulo, Brazil \\ ${ }^{2}$ Departamento de Física de Partículas \& Instituto Galego de Física de Altas Enerxías, Univ. de Santiago \\ de Compostela, 15782 Santiago de Compostela, Spain
}

\begin{abstract}
.
Traditionally, the depth of maximum shower development $X_{\max }$ has been used as a surrogate observable for composition. Here we present the possibility of a new methodology to discriminate between light and heavy cosmic-ray primaries on an event-by-event basis. This method is based on comparisons between detected radio signals and Monte Carlo simulations, but instead of first reconstructing $X_{\max }$, we try to infer the cosmic-ray composition directly. We show that a large discrimination efficiency could in principle be reached for zenith angles above $\theta \simeq 65^{\circ}$, even when some of the typical uncertainties in radio detection are taken into account.
\end{abstract}

\section{Introduction}

Radio detection has been shown to be able to reconstruct both energy [1] and $X_{\max }[2,3]$ with comparable uncertainties to fluorescence detectors in the case of low zenith angle showers $\left(\theta \leq 55^{\circ}\right)$ and with an almost $100 \%$ duty cycle. The first radio-based reconstruction of $X_{\max }$ [4] is based on comparisons between the measured electric fields and scintillator data with simulations of proton and iron showers.

In this work we propose to bypass the $X_{\max }$ reconstruction and infer the composition of an event directly, by comparing its radio footprint with the ones from simulated proton and iron showers.

\section{Inferring primary composition on an event-by-event basis}

To determine the primary composition of a shower event, we firstly perform ZHAIRES Monte Carlo simulations of 50 proton and 50 iron-initiated showers with the same energy and geometry of the input event. The measured peak of the radio signal at each antenna $\vec{E}_{\text {data }}$ is then compared with the peak electric field obtained from simulations $\vec{E}_{\mathrm{MC}}$ to calculate $\Delta_{s}^{2}$ (Eq. 1), where $f_{s}$ is an energy scaling factor (see below), $\vec{r}_{\text {core }}=\left(x_{\text {core }}, y_{\text {core }}\right)$ is the position of the shower core in the simulation and $(x, y)$ is the position of each antenna. The sums run over the three components of the peak electric field and over all the antennas with signal. To account for uncertainties in both the detected energy and core position, the position of the core $\left(\vec{r}_{\text {core }}\right)$ and the energy scaling factor $\left(f_{s}\right)$ can be varied ${ }^{1}$, leading to

^e-mail: carvajr@gmail.com

${ }^{1}$ In this work we did not take into account an uncertainty in energy, so a fixed value $f_{s}=1$ was used in the minimization. Also, cosmic-ray energy reconstruction is not performed, but as shown in [1], using the radio technique it can be obtained with an accuracy of $\sim 17 \%$. Events with 3 or more antennas tend to be better reconstructed. 
different values of $\Delta_{s}^{2}\left(f_{s}, \vec{r}_{\text {core }}\right)$ for each simulation. But only the minimum value (denoted simply as $\Delta^{2}$ ) is used in the reconstruction, corresponding to the values of $f_{s}$ and $\vec{r}_{\text {core }}$ for which the simulated shower best represents the measured event.

$$
\Delta_{s}^{2}=\sum_{i}\left(\sum_{\text {all antennas }}\left[E_{i, \text { data }}-f_{s} \cdot E_{i, M C}\left(x_{\text {ant }}-x_{\text {core }}, y_{\text {ant }}-y_{\text {core }}\right)\right]^{2}\right)
$$

In this discrimination method $X_{\max }$ is not determined, instead we compare the distributions of $\Delta^{2}$ obtained for each simulated composition and infer the most likely composition of the detected event directly. For that purpose, and for the sake of simplicity, we compare the averages of $\Delta^{2}$ obtained using the proton ${ }^{2}$ and iron simulations. The detected event is classified as proton (light) if $\left\langle\Delta^{2}\right\rangle_{p} \leq\left\langle\Delta^{2}\right\rangle_{F e}$ or iron (heavy) if $\left\langle\Delta^{2}\right\rangle_{p}>\left\langle\Delta^{2}\right\rangle_{F e}$, i.e. the event is classified as proton or iron depending on what type of primary, on average, induces electric fields that are more similar to the detected fields.

We have included some factors that affect the measurement of radio emission: noise, (galactic) background and uncertainties in the position of the reconstructed shower core ${ }^{3}$. We have not taken into account energy and arrival direction uncertainties (see also Section 3). On the top panel of Fig. (1) we show an example of the classification procedure for an input proton shower with $E=10^{18} \mathrm{eV}$ and $\theta=65^{\circ}$, triggering a squared-grid array with distance between antennas $D=750 \mathrm{~m}$. On the bottom left panel of Fig. (1) we show our results for several zenith angles. One can see that the method is most efficient for zenith angles $\theta>60^{\circ}$, the region where the uncertainties on $X_{\max }$ for methods similar to the LOFAR one increase sharply ${ }^{4}$ (see bottom right panel of Fig. (1)). All simulations used showers of energy $10^{18} \mathrm{eV}$, taken as an intermediate energy to illustrate the performance of the method. More details can be seen in [5].

\section{Discussion and conclusion}

The complexity of the radio emission footprint and its dependence on shower geometry and sensitivity to the whole shower longitudinal profile (and not only to $X_{\max }$ ) is responsible for the ability to distinguish between light and heavy primaries, even if the showers have very similar $X_{\max }$. In this type of event, the $\Delta^{2}$ variable allows a better separation than $X_{\max }$, as can be seen on the top left panel of Fig. (1), where even for very similar values of $X_{\max }$ (e.g. $X_{\max } \sim 680 \mathrm{~g} / \mathrm{cm}^{2}$ ), proton and iron primaries have significantly different $\Delta^{2}$.

Up to now we have not taken into account uncertainties in shower energy, which could in principle pose a problem to the discrimination method. To investigate this, we allowed $f_{s}$ in Eq. (1) to vary freely in the minimization of our set of showers at $65^{\circ}$. By doing this, our method experienced only a small decrease in overall efficiency, from $\sim 90 \%$ to $\sim 80 \%$. We defer a more in-depth study of the influence of energy uncertainties on the discrimination efficiency to a later study.

We have proposed a new methodology to infer the nature of cosmic-ray primaries based on comparisons between detected radio signals and multiple simulations. This methodology bypasses the

\footnotetext{
${ }^{2}$ It is well known that the fluctuations of many observables $\left(X_{\max }, N_{\max }\right.$, etc..) in proton-induced showers are larger than in iron-induced showers. This naturally translates itself into a larger spread of $\Delta^{2}$ for $\mathrm{p}$ showers, which could cause a bias in the method. To avoid this, we do not consider simulations that have very different footprints from the one in the detected event. This is accomplished by a cut that removes simulations with $\Delta^{2}$ above a certain cut value $\Delta_{\max }^{2}$ before calculating the averages.

${ }^{3}$ We estimated a very generous upper limit to the effect of noise on the measured peak electric field as a Gaussian with $\sigma_{\text {noise }}=30 \mu \mathrm{V} / \mathrm{m}$ per component of the electric field. An electric field background with a fixed amplitude of $3 \mu \mathrm{V} / \mathrm{m}$ and a shift in the core position $\vec{P}_{\text {error }}=\left(r_{\text {error }}, \varphi_{\text {error }}\right)$, with $\sigma_{r_{\text {error }}}=50 \mathrm{~m}$, were also included. In general we have an error distribution of less than $25 \mathrm{~m}$ in the reconstructed core position after the minimization, even at the highest zenith angles.

${ }^{4}$ We estimated the intrinsic uncertainty on $X_{\max }$ using a simplified version of the LOFAR method [5].
} 

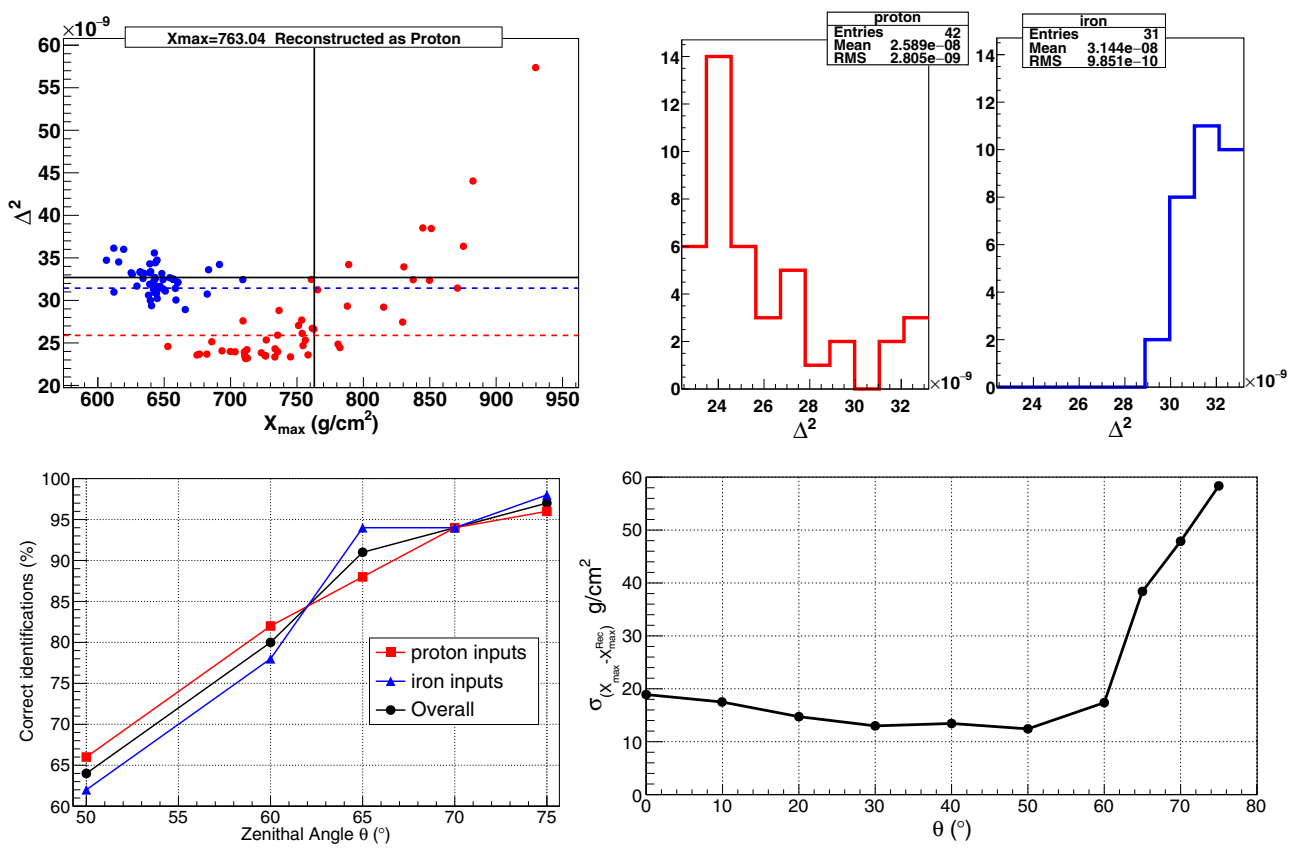

Figure 1. Top: Example of the classification for an input proton shower. Top left: $\Delta^{2}$ vs $X_{\max }$ obtained from each simulation ( $\mathrm{p}$ in red, Fe in blue). The red and blue dashed lines represent $\left\langle\Delta^{2}\right\rangle_{p}$ and $\left\langle\Delta^{2}\right\rangle_{F e}$, respectively. Top right: $\Delta^{2}$ distributions. Bottom Left: Fraction of correct identifications of the nature of the primary initiating the event for each $\theta$. Bottom right: Intrinsic uncertainty on $X_{\max }^{\mathrm{Rec}}$ as a function of $\theta$ over a square array with $\mathrm{D}=500 \mathrm{~m}$, without taking into account any detection uncertainties.

reconstruction of $X_{\max }$ and is, in principle, capable of discriminating between light and heavy primary compositions on an event-by-event basis with an efficiency of $80 \%$ (90\%) for zenithal angles above $60^{\circ}\left(65^{\circ}\right)$.

\section{Acknowledgments}

W.R.C. is supported by Grant 2015/15735-1, São Paulo Research Foundation (FAPESP). J.A.-M. thanks Ministerio de Economía (FPA 2017-85114-P), Xunta de Galicia (ED431C 2017/07), Feder Funds, 7th Framework Program (PIRSES-2009-GA-246806) RENATA Red Nacional Temática de Astropartículas (FPA2015-68783-REDT) and Maria de Maeztu Unit of Excellence MDM-2016-0692.

\section{References}

[1] A. Aab et al. (The Pierre Auger Collaboration), Phys. Rev. D 93, 122005 (2016)

[2] J. R. Hörandel et al., arXiv:1705.04233 [astro-ph.HE]

[3] F. Gaté, for the Pierre Auger Collaboration, arXiv:1702.02905 [astro-ph.HE]

[4] S. Buitink et al., Phys. Rev. D 90, 082003 (2014)

[5] W. Carvalho Jr and J. Alvarez-Muñiz, arXiv:1712.03544 [astro-ph.HE] 